\title{
Switching Retinogeniculate Axon Laterality Leads to Normal Targeting but Abnormal Eye-Specific Segregation That Is Activity Dependent
}

\author{
Alexandra Rebsam, ${ }^{1,2}$ Timothy J. Petros, ${ }^{1,2}$ and Carol A. Mason ${ }^{1,2,3}$ \\ Departments of ${ }^{1}$ Pathology and Cell Biology, ${ }^{2}$ Neuroscience, and ${ }^{3}$ Ophthalmology, College of Physicians and Surgeons, Columbia University, New York, \\ New York 10032
}

Partial decussation of sensory pathways allows neural inputs from both sides of the body to project to the same target region where these signals will be integrated. Here, to better understand mechanisms of eye-specific targeting, we studied how retinal ganglion cell (RGC) axons terminate in their thalamic target, the dorsal lateral geniculate nucleus (dLGN), when crossing at the optic chiasm midline is altered. In models with gain- and loss-of-function of $\mathrm{EphB1}$, the receptor that directs the ipsilateral projection at the optic chiasm, misrouted RGCs target the appropriate retinotopic zone in the opposite dLGN. However, in EphB1 ${ }^{-1-}$ mice, the misrouted axons do not intermingle with normally projecting RGC axons and segregate instead into a distinct patch. We also revisited the role of retinal activity on eye-specific targeting by blocking correlated waves of activity with epibatidine into both eyes. We show that, in wild-type mice, retinal waves are necessary during the first postnatal week for both proper distribution and eye-specific segregation of ipsilateral axons in the mature dLGN. Moreover, in $E \mathrm{PhB1} 1^{-1-}$ mice, refinement of ipsilateral axons is perturbed in control conditions and is further impaired after epibatidine treatment. Finally, retinal waves are required for the formation of the segregated patch of misrouted axons in $E p h B 1^{-1-}$ mice. These findings implicate molecular determinants for targeting of eye-specific zones that are independent of midline guidance cues and that function in concert with correlated retinal activity to sculpt retinogeniculate projections.

\section{Introduction}

Many brain regions receive diverse inputs that must be precisely organized to properly integrate and transmit neural information. Some relay regions receive inputs from both sides of the body through partial decussation of axonal projections at the midline. The precise ratio of ipsilateral and contralateral inputs is crucial for appropriate targeting and sensory or motor function (Victor et al., 2000; Kullander et al., 2003; Jen et al., 2004; Kaas, 2005; Beg et al., 2007; Iwasato et al., 2007; Wegmeyer et al., 2007).

In the visual system, the partial decussation of retinal ganglion cell (RGC) axons at the optic chiasm midline ensures that targets on each side of the brain receive inputs from both eyes to implement binocular vision. In the mouse, retinal axons map topographically onto their thalamic target, the dorsal lateral geniculate nucleus (dLGN), guided by EphA and ephrin-A gradients in the retina and the dLGN (Feldheim et al., 1998; Pfeiffenberger et al., 2005). Retinal axons from both eyes

\footnotetext{
Received July 17, 2009; revised 0ct. 2, 2009; accepted 0ct. 16, 2009.

This work was supported by National Institutes of Health Grants EY012736 and EY015290, the Gatsby Initiative in Brain Circuitry (C.A.M.), Fondation pour la Recherche Medicale, and the Human Frontier Science Program (A.R.). We thank Ray Guillery, Wesley Grueber, John Martin, Samit Chakrabarty, Pierre Godement, Takeshi Sakurai, and members of the Mason Laboratory for helpful discussions and critical review of this manuscript.

Correspondence should be addressed to Carol A. Mason, Departments of Pathology and Cell Biology, and Neuroscience, College of Physicians and Surgeons, PS 14-509, Columbia University, 630 West 168th Street, New York, NY 10032.E-mail: cam4@columbia.edu.

T. J. Petro's present address: Weill Cornell Medical College, New York, NY 10065. DOI:10.1523/JNEUROSCI.3462-09.2009

Copyright $\odot 2009$ Society for Neuroscience $\quad$ 0270-6474/09/2914855-09\$15.00/0
}

segregate from an initially overlapping innervation pattern into distinct territories (Godement et al., 1984; Jaubert-Miazza et al., 2005). The molecular factors that mediate eye-specific innervation are not well understood (Leamey et al., 2007), but it is widely accepted that molecular directives in concert with retinal activity influence the final pattern of eye-specific innervation within thalamic targets (Chalupa, 2007; Huberman et al., 2008a).

RGCs that project ipsilaterally originate in the ventrotemporal (VT) retina and express the transcription factor Zic2, which in turn regulates expression of the guidance receptor EphB1 (Herrera et al., 2003; Williams et al., 2003; García-Frigola et al., 2008; Lee et al., 2008). EphB1-expressing VT retinal growth cones are repulsed by ephrin-B2-expressing glia at the optic chiasm midline, thereby establishing the ipsilateral projection (Williams et al., 2003). RGCs that project contralaterally express the transcription factor Islet2 (Pak et al., 2004) and the adhesion molecule NrCAM $\left[\mathrm{N}_{\mathrm{g}}\right.$ (neuron-glia) related cell adhesion molecule CAM-] (Williams et al., 2006).

We investigated whether decussation errors at the optic chiasm affect topographic targeting in the dLGN, by altering one of the molecular determinants for laterality. We examined models in which EphB1 expression is modified: gain-of-function of EphB1 via in utero retinal electroporation increases the number of ipsilaterally projecting fibers (Petros et al., 2009), whereas loss of EphB1 in EphB1 $1^{-1-}$ mice results in a decreased ipsilateral projection (Williams et al., 2003) with more VT RGCs projecting contralaterally. We asked the following: (1) Do misrouted RGC axons terminate in retinotopically appropriate zone in the oppo- 
site dLGN? (2) Do misrouted RGC axons terminate in zones that are distinct from those of normally routed axons? (3) How does retinal activity affect eye-specific innervation when ipsilateral and contralateral inputs are imbalanced?

We find that aberrations in decussation do not impair RGC targeting in the dLGN. However, misrouted axons abnormally segregate from their normal counterparts. In addition, the refinement of the normal ipsilateral projection is impaired in $E p h B 1^{-1-}$ mice, and retinal activity is crucial for the proper distribution and segregation of both normal and misrouted axons. These experiments provide insight into how the ratio of ipsilateral and contralateral axons, in concert with retinal activity, affect eye-specific retinogeniculate targeting.

\section{Materials and Methods}

Animals. Wild-type C57BL/6J mice and EphB1 ${ }^{-1-}$ mice maintained on C57BL/6J background (Williams et al., 2003) were maintained in a timed-pregnancy breeding colony at Columbia University. C57BL/6J mice were used for all retinal in utero electroporations. Embryonic day 0 (E0) was defined as midnight of the night before a plug was found, and postnatal day $0(\mathrm{P} 0)$ is the day of birth. All animal procedures followed the regulatory guidelines of the Columbia University Institutional Animal Care and Use Committee.

Anterograde labeling of retinogeniculate projections. P7, P13, and P30 mice were anesthetized with ketamine-xylazine (100 and $10 \mathrm{mg} / \mathrm{kg}$, respectively, in $0.9 \%$ saline). The skin was cut open at the eyelid junction. Eyes were injected with a glass micropipette intravitreally with $1-5 \mu \mathrm{l}$ of $0.2 \%$ cholera toxin subunit B (CTB) conjugated to Alexa Fluor 594, 488 , or 647 (Invitrogen) diluted in 1\% DMSO. In some P13 pups, small volumes of liquid DiI $(0.4 \%$ in $4 \%$ dimethylformamide and $10 \%$ sucrose) or CTB-Alexa Fluor 594 (0.1\% in 0.5\% DMSO, 10\% sucrose solution) were injected into the VT retina using a Picospritzer II. After 1-2 d, mice were anesthetized and perfused transcardially with $4 \%$ paraformaldehyde in $0.1 \mathrm{~m}$ phosphate buffer before dissection of brain and retinas. Brains were postfixed overnight, embedded in $3 \%$ agarose, and sectioned with a vibratome $(100 \mu \mathrm{m})$. Sections were processed for immunohistochemistry or directly mounted in Gelmount.

In utero retinal electroporation. The procedure for in utero retinal electroporation has been previously described (García-Frigola et al., 2007; Petros et al., 2009). Briefly, a DNA solution $(0.8 \mu \mathrm{g} / \mu \mathrm{l}$ pCAG-GFP plasmid plus or minus $2-5 \mu \mathrm{g} / \mu \mathrm{l}$ pCAG-HA-EphB1 plasmids plus $0.03 \%$ fast green) was injected using a pulled glass micropipette into one eye of each E14.5 embryo, and then electroporated with five $40 \mathrm{~V}$ pulses. Dams were allowed to give birth naturally. At P7, retinogeniculate projections were anterogradely labeled and processed as described above. Fixed retinas were assessed for green fluorescent protein (GFP) labeling under a fluorescent dissecting microscope (Zeiss) before retina and brain section immunostaining. Cases with very little GFP staining in the retina or with GFP staining in VT retina were not used for analysis.

Epibatidine injection. Postnatal mice were anesthetized on ice. The skin was cut open at the eyelid junction to access the eye. A total of $0.5-1 \mu \mathrm{l}$ of $1 \mathrm{~mm}$ epibatidine in $0.9 \%$ saline was injected intravitreally in both eyes through the same injection site every $24 \mathrm{~h}$ from P3 to P6. Pups were returned to their mother after recovery from anesthesia.

Immunostaining. Ten percent normal donkey serum in $0.3 \%$ Triton was used as blocking solution. GFP was detected using a rabbit anti-GFP antibody (1/1000; Invitrogen) and a donkey anti-rabbit Alexa 488 secondary antibody (1/400; Invitrogen). For the retinas, HA-EphB1 was detected using a rat anti-HA antibody (1/500; Roche) and a donkey anti-rat Cy3 (1/500) or Cy5 (1/250) secondary antibody (Jackson ImmunoResearch). Retinas were flat-mounted and brain sections mounted in Gelmount.

Microscopy. Imaging retinal whole mounts and brain sections was done with Axiovision or OpenLab software through Axiophot Camera and a Zeiss Axioplan 2 microscope (for $E p h B 1^{-1-}$ and wild-type mice) or with a Zeiss LSM 510 confocal microscope (for electroporated mice).
$Z$ stacks were taken and projected into two-dimensional image using the LSM software projection tool.

Analysis of the number, distribution, and segregation of ipsilateral fibers. Quantification of eye-specific segregation in Figures 3 and 5 was performed on $10 \times$ images obtained with Zeiss Axioplan fluorescence microscope from three consecutive coronal sections through the region of the dLGN containing the greatest extent of the ipsilateral projection. Quantification of eye-specific segregation was performed also on $20 \times$ images obtained with Zeiss LSM 510 confocal microscope [laser power, 80\%; gain, 370 (red channel) and 400 (green channel); offset, -0.08 (red channel) and -0.21 (green channel)], on one section per case (the middle section in the three sections used for the quantification at $10 \times$ magnification) (supplemental Fig. S3, available at www.jneurosci.org as supplemental material). For both magnifications, we have used variable thresholds for the contralateral projection and a fixed threshold for the ipsilateral projection. As Alexa Fluor 594-labeled contralateral/Alexa Fluor 488-labeled ipsilateral projections had a better signal-to-noise ratio, we conducted our analyses on these images. Using MetaMorph software (Molecular Devices), the boundary of the dLGN was outlined, excluding the intrageniculate leaflet, the ventral lateral geniculate nucleus, and the optic tract (supplemental Fig. S2d, available at www. jneurosci.org as supplemental material) in $10 \times$ images and using the entire image at $20 \times$ magnification. The intensity threshold for the ipsilateral projection was chosen when the signal-to-background ratio was at least 1.2 (see Fig. 4; supplemental Fig. S2c, available at www.jneurosci.org as supplemental material), especially at the border of the ipsilateral patch, or around the scattered ipsilateral fibers. In general, this value was between 50 and 80 for $10 \times$ magnification and 35 and 45 for $20 \times$ magnification images (grayscale span from 0 to 255). The overlap between ipsilateral and contralateral projections (pixel overlap) was measured at every 10th threshold value for the contralateral image to obtain the function of overlap between ipsilateral and contralateral projections (see Figs. $3 b, 5 c, d$ ), as previously described (Muir-Robinson et al., 2002; Torborg and Feller, 2004). The proportion of dLGN occupied by ipsilateral axons was measured as a ratio of ipsilateral pixels to the total number of pixels in the dLGN region. The extent of eye-specific projections within the dLGN along the dorsoventral (DV) and mediolateral (ML) axes of the dLGN was measured by tracing a line along the DV or ML axis of the dLGN, tilted compared with the real DV and ML axes of the section (supplemental Fig. S2a, available at www.jneurosci.org as supplemental material). The extent of the ipsilateral projection was measured by tracing a line between the two points delineating the maximal extent of the ipsilateral signal along the chosen axis (supplemental Fig. S2a,g, $h$, available at www.jneurosci.org as supplemental material). The extent of the ipsilateral projection along the dLGN axis is calculated by dividing the length of the ipsilateral projection by the dLGN length and is expressed as a percentage of dLGN length. Statistical analyses were performed using a Student's $t$ test. As no significant differences were found between C57BL/ 6J, EphB1 $1^{+/-}$and EphB1 ${ }^{+/+}$(ANOVA test), the results of these genotypes were pooled and designated as wild-type mice.

\section{Results}

After ectopic expression of EphB1, retinal axons from non-VT retina are misrouted ipsilaterally but still target the contralateral input-recipient region of the dLGN

We recently reported that when GFP and HA-EphB1 are coelectroporated into non-VT RGCs at E14.5, the proportion of ipsilateral GFP + fibers is increased by sevenfold (from 3 to 22\%) compared with electroporation of GFP alone (Petros et al., 2009). Here, we use a similar gain-of-function approach to assess how RGCs that ectopically express EphB1 and aberrantly project ipsilaterally, are distributed within the dLGN. At P8, GFP+ RGC axons were clearly visible in the central retina (Fig. 1a), optic chiasm (Fig. 1b), and dLGN (Fig. $1 d-g$ ). Anterograde labeling of the entire retinal projection from each eye at P8 (Fig. 1c) confirmed that the segregation of eye-specific fibers remains intact after electroporation: ipsilateral axons form a core in the dorso- 


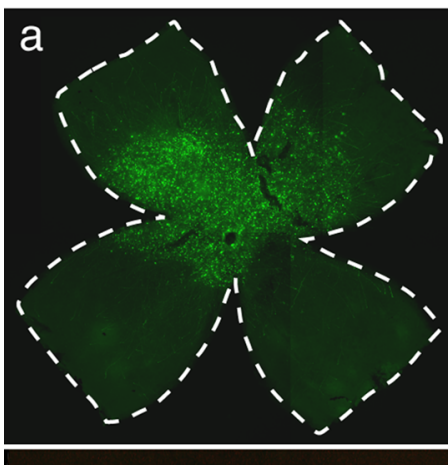

b
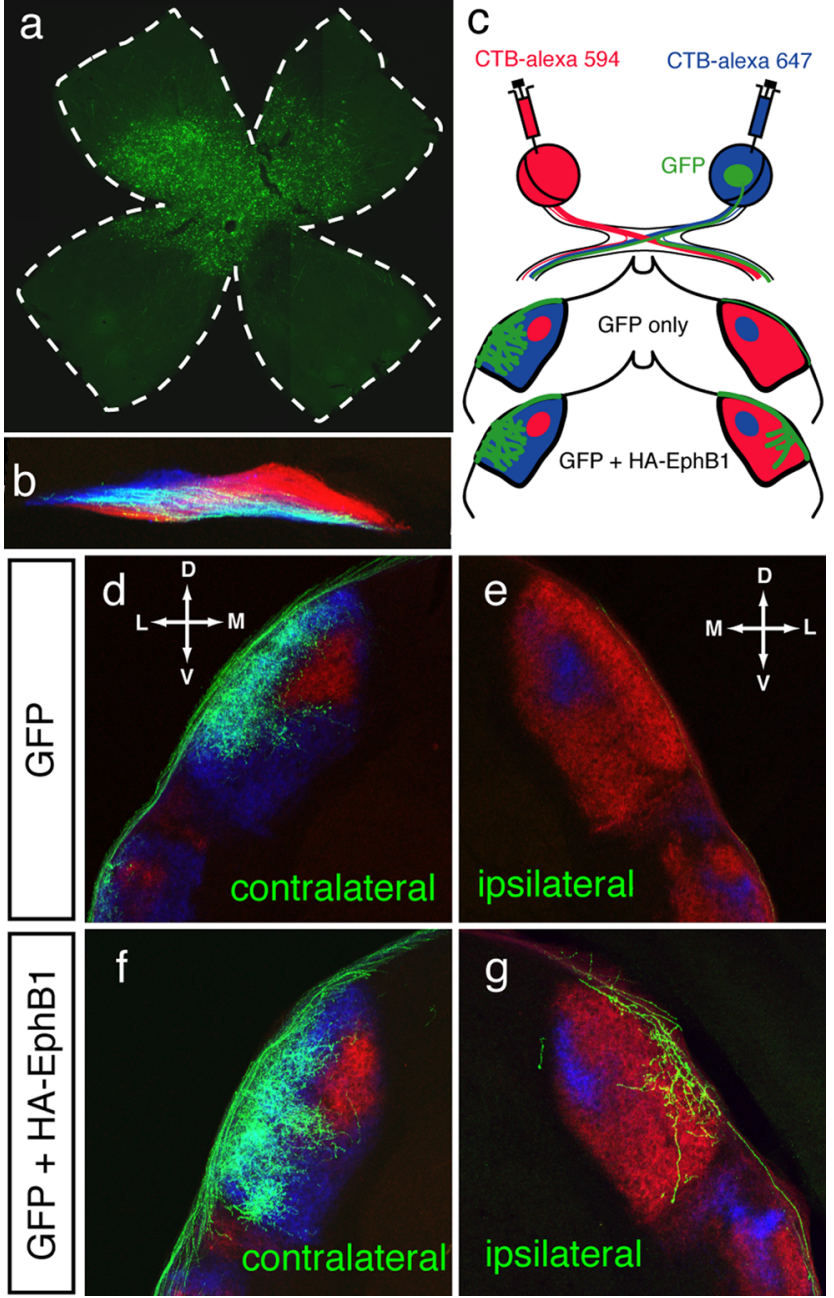

Figure 1. Retinogeniculate targeting at $\mathrm{P} 8$ after in utero retinal electroporation of EphB1 constructs at E14.5. $\boldsymbol{a}$, Retinal flat mount indicating GFP expressing RGCs in central retina. $\boldsymbol{b}$, $\mathrm{GFP}+\mathrm{RGC}$ fibers at the optic chiasm. Retinal axons from each eye are in blue and red (see $c$ ) and GFP + axons appear white. c, Scheme depicting the whole-eye labeling with CTB-Alexa Fluor dyes and GFP labeling along the retinogeniculate pathway. In GFP control electroporations, most GFP + fibers project to the contralateral dLGN (d), with few (if any) GFP + fibers projecting ipsilaterally $(\boldsymbol{e})$. After GFP + HA-EphB1 electroporations $(\boldsymbol{f}, \boldsymbol{g})$, there is an increase in GFP + fibers in the ipsilateral dLGN. Both contralateral $(\boldsymbol{f})$ and ipsilateral $(\boldsymbol{g}) \mathrm{GFP}+$ fibers project to the appropriate retinotopic region in the contralateral-recipient region ( $g$ ). D, Dorsal; V, ventral; $M$, medial; L, lateral.

medial region of the dLGN and are surrounded by contralateral axons (Fig. 1d-g).

In GFP-only electroporated cases $(n=9)$, most fibers project contralaterally to the outer shell of the dLGN (Fig. 1d). Very few or no GFP + fibers were found in the ipsilateral optic tract and $\mathrm{dLGN}$ at $\mathrm{P} 8$ (Fig. 1e). Although most GFP+ fibers remain contralateral (Fig. $1 f)$ after GFP + EphB1 electroporations $(n=13)$, an increased ipsilateral GFP+ projection is apparent (Fig. $1 g$ ). These results are in agreement with those by Petros et al. (2009) and confirm that EphB1 is able to reroute some non-VT RGCs ipsilaterally.

In both control and EphB1 electroporated cases, GFP+ retinal fibers that maintain a contralateral trajectory project into and arborize within the contralateral axon-recipient region of the dLGN (Fig. 1d,f). Notably, GFP + fibers that are misrouted ipsilaterally after electroporation of EphB1, project to the contralateral axon-recipient region of the dLGN and not to the ipsilateral

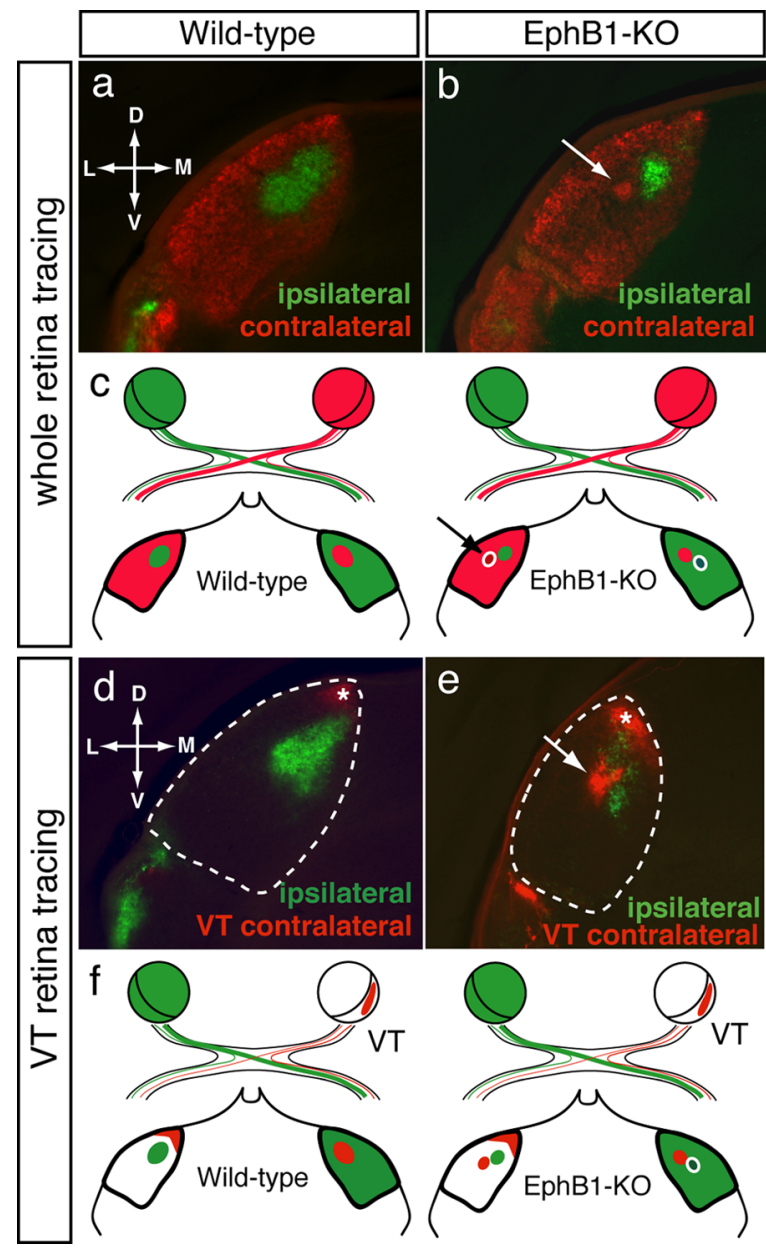

Figure 2. Retinogeniculate projections at P31 in wild-type and EphB1 ${ }^{-1-}$ mice. Coronal sections through the dLGN after whole-eye tracing in one eye and either whole eye $(\boldsymbol{a}, \boldsymbol{b})$ or VT retina $(\boldsymbol{d}, \boldsymbol{e})$ anterograde tracing from the other eye. $\boldsymbol{a}, \boldsymbol{d}$, In wild-type mice, the ipsilateral projection (green) forms a patch in the dorsocentral dLGN surrounded by contralateral terminations (red). Late-born VT RGCs project contralaterally to the dorsal tip of the dLGN (d, e, asterisk). $\boldsymbol{b}, \boldsymbol{e}, \ln E p h B 1^{-1-}$ mice, an ectopic patch (arrow) arising from the contralateral eye is adjacent to, but segregated from, the reduced ipsilateral patch. VT RGCs project contralaterally to two distinct sites $(\boldsymbol{e})$ : the dorsal tip of the dLGN, corresponding to the "normal" VT contralateral projection (asterisk), and the ectopic patch (arrow). c, $\boldsymbol{f}$, Schemes depict dLGN termination regions after the anterograde tracing paradigms in wild-type and EphB1 ${ }^{-1-}$ mice. D, Dorsal; $\mathrm{V}$, ventral; $M$, medial; L, lateral.

axon-recipient region (Fig. 1g). Thus, ectopic EphB1-expressing RGCs in non-VT retina are misrouted ipsilaterally and target the region corresponding to their origin in the retina, independent of their decussation pattern at the optic chiasm midline.

In EphB1 ${ }^{-1-}$ mice, VT RGC axons are misrouted contralaterally and form a distinct patch in the ipsilateral axon-recipient zone of the dLGN.

To further study the relationship of guidance decisions at the chiasm midline and eye-specific targeting in the dLGN, we examined RGC projections to the dLGN in $E p h B 1^{-1-}$ mice that have a severely reduced ipsilateral projection (Williams et al., 2003). As EphB1 is not expressed in the dLGN during the period of innervation and segregation (supplemental Fig. S1, available at www. jneurosci.org as supplemental material), the loss of EphB1 function is not likely to induce changes in the dLGN itself.

Using whole-eye labeling, we compared RGC targeting in the wild-type and EphB1 ${ }^{-1-}$ dLGN (Fig. $2 a-c$ ). In P31 wild-type mice $(n=10)$, the ipsilateral projection from the VT retina forms 
a distinct patch in the dorsocentral dLGN and is surrounded by the contralateral projection (Fig. $2 a$ ), in agreement with previous reports (Godement et al., 1984; Jaubert-Miazza et al., 2005). In $E p h B 1^{-l-}$ mice $(n=8)$, the reduced projection from the ipsilateral eye is correctly positioned within the dorsocentral dLGN but is smaller than wild type (Fig. $2 b$ ).

Strikingly, in the dLGN of P31 EphB1 ${ }^{-/-}$mice, a segregated patch of contralateral retinal axons is positioned adjacent to the normal ipsilateral input recipient region (note the intensity of fibers within and the reduced density of fibers around this patch) (Fig. $2 b$ ). To test whether this segregated patch in the EphB1 ${ }^{-1-}$ mice arises from contralaterally misrouted VT RGCs, we labeled one entire retina with CTB-Alexa Fluor 488 and the VT retina of the opposite eye with DiI (or CTB-Alexa Fluor 594) at P13 (Fig. $2 f$ ). In wild-type mice, VT RGCs project ipsilaterally to the dorsocentral dLGN and contralaterally to the dorsal tip of the dLGN (Fig. 2d) (Reese and Cowey, 1987; Pfeiffenberger et al., 2006). In EphB1 ${ }^{-1-}$ mice, VT RGCs display an additional projection to the dLGN (Fig. 2e), corresponding to the ectopic patch seen after whole-eye labeling (Fig. $2 b$ ). Thus, this projection pattern confirms that the ectopic patch in EphB1 $1^{-/-}$dLGN arises from VT RGCs that are misdirected to the contralateral side and retain their topography, but segregate from other retinal projections (Figs. 2f, 6). In conclusion, gain- and loss-of-function experiments with EphB1 demonstrate that altering guidance decisions at the midline does not affect targeting of RGC axons but that contralaterally misrouted axon terminations form a distinct patch that does not mingle with the normal projections.

Refinement of ipsilateral retinal axons to the dLGN is impaired in EphB1 ${ }^{-/-}$mice

In wild-type mice, eye-specific territories are completely segregated by P31 (Fig. 2a) (Jaubert-Miazza et al., 2005). We specifically examined whether the reduction in ipsilateral inputs observed in EphB1 ${ }^{-1-}$ mice influences the distribution and segregation of the remaining ipsilateral inputs (Fig. 3). In EphB1 ${ }^{-l-}$ mice, the proportion of ipsilateral fibers in the dLGN is reduced by $84 \%$ compared with wild-type mice (wild type, $17.6 \%$, and EphB1-KO, 2.9\% of dLGN territory is occupied by ipsilateral fibers; $p=8.5 \mathrm{E}-12$ ). Furthermore, the extent of the ipsilateral projection is significantly reduced along the DV and ML axes (Fig. 3a).

EphB1 ${ }^{-1-}$ mice also display defects in eye-specific segregation, as there is significant overlap between ipsilateral and contralateral fibers compared with wild type at P31 (Fig. 3b). Thus, both eye-specific distribution and segregation are perturbed in EphB1 ${ }^{-1-}$ mice.

\section{Alterations in retinal activity further disrupts RGC fiber distribution and segregation in the dLGN}

Neural activity in the retina is important for eye-specific segregation (Chalupa, 2007; Huberman et al., 2008a). As spontaneous waves of retinal activity rely on cholinergic transmission through the first postnatal week, ocular injections of the nicotinic acetylcholine receptor agonist epibatidine decorrelates RGC activity and eliminates retinal waves (Penn et al., 1998; Sun et al., 2008b). During the second postnatal week, retinal waves continue but are cholinergic-independent and glutamatergic-dependent (Bansal et al., 2000; Wong et al., 2000; Zhou and Zhao, 2000).

Given the changes observed in EphB1 ${ }^{-1-}$ mice in the proportion, distribution, and segregation of, in particular, the ipsilateral RGC axons, we examined whether retinal activity is required during the first postnatal week for (1) the segregation of the normal
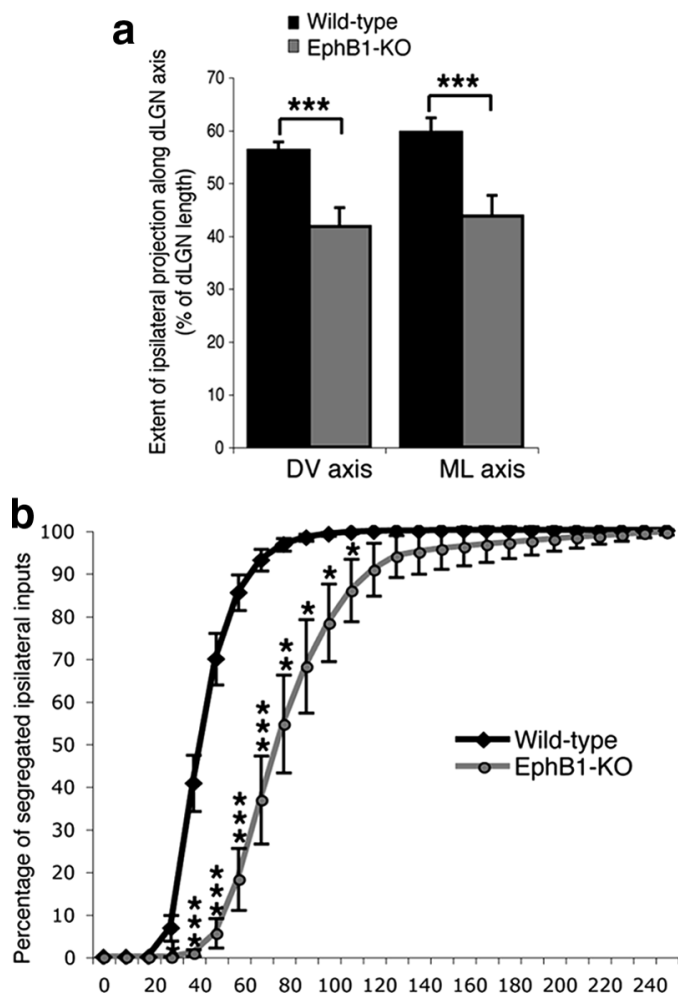

Figure 3. Distribution and segregation of ipsilateral retinal projections in wild-type and EphB1 ${ }^{-1-}$ mice. $\boldsymbol{a}$, Extension of the ipsilateral projection expressed as a percentage of length along the DV and ML axes of the dLGN. Ipsilateral fibers cover less territory along the DV and ML dLGN axes in EphB1 ${ }^{-1-}$ mice compared with wild-type mice. $\boldsymbol{b}$, Segregation plot. Percentage of pixels containing only ipsilateral signal (no contralateral signal) as a function of contralateral threshold (ipsilateral threshold is fixed). In EphB1 ${ }^{-1-}$ mice, ipsilateral fibers are less segregated from contralateral fibers (more overlap) than in wild-type mice. Error bars indicate SEM values. ${ }^{*} p<0.05,{ }^{* *} p<0.01,{ }^{* * *} p<0.001$.

ipsilateral and contralateral components, and (2) the segregation of misrouted VT contralateral axons in EphB1 ${ }^{-1-}$ mice.

We examined these features at P31, after binocular epibatidine treatment from P3 to P6. Previous reports have focused on the short-term effects of epibatidine treatment at P9, without assessing the long-term effects on retinogeniculate projection after glutamatergic-dependent retinal waves have ended (Rossi et al., 2001; Pfeiffenberger et al., 2005). We report that blocking retinal waves during the first postnatal week has lasting effects on the distribution and segregation of ipsilateral axons in both wildtype and EphB1 ${ }^{-1-}$ mice.

After epibatidine treatment of wild-type mice $(n=7)$, ipsilateral fibers were distributed over a larger territory along the DV and ML axes compared with saline-treated wild-type mice $(n=5)$ (Figs. $4 a, b, 5 b)$. Because the proportion of dLGN occupied by ipsilateral fibers is not significantly increased (Fig. 5a), the expanded distribution of ipsilateral fibers reflects the scattering of ipsilateral arbors observed after epibatidine treatment (supplemental Fig. S2, available at www.jneurosci. org as supplemental material; Fig. 7). In addition, ipsilateral fibers are less segregated from contralateral fibers after epibatidine treatment (Figs. $4 b, 5 c, d$ ).

In contrast, in EphB1 ${ }^{-1-}$ mice (Fig. $4 c, d$ ), epibatidine treatment ( $n=11$ ) reduces the percentage of dLGN territory occupied by ipsilateral fibers compared with saline-treated $E p h B 1^{-1-}$ mice $(n=5)$ (Figs. $4 c, d, 5 a)$. However, there is a concomitant increase in the extent of ipsilateral fibers along the DV and ML axes of the 


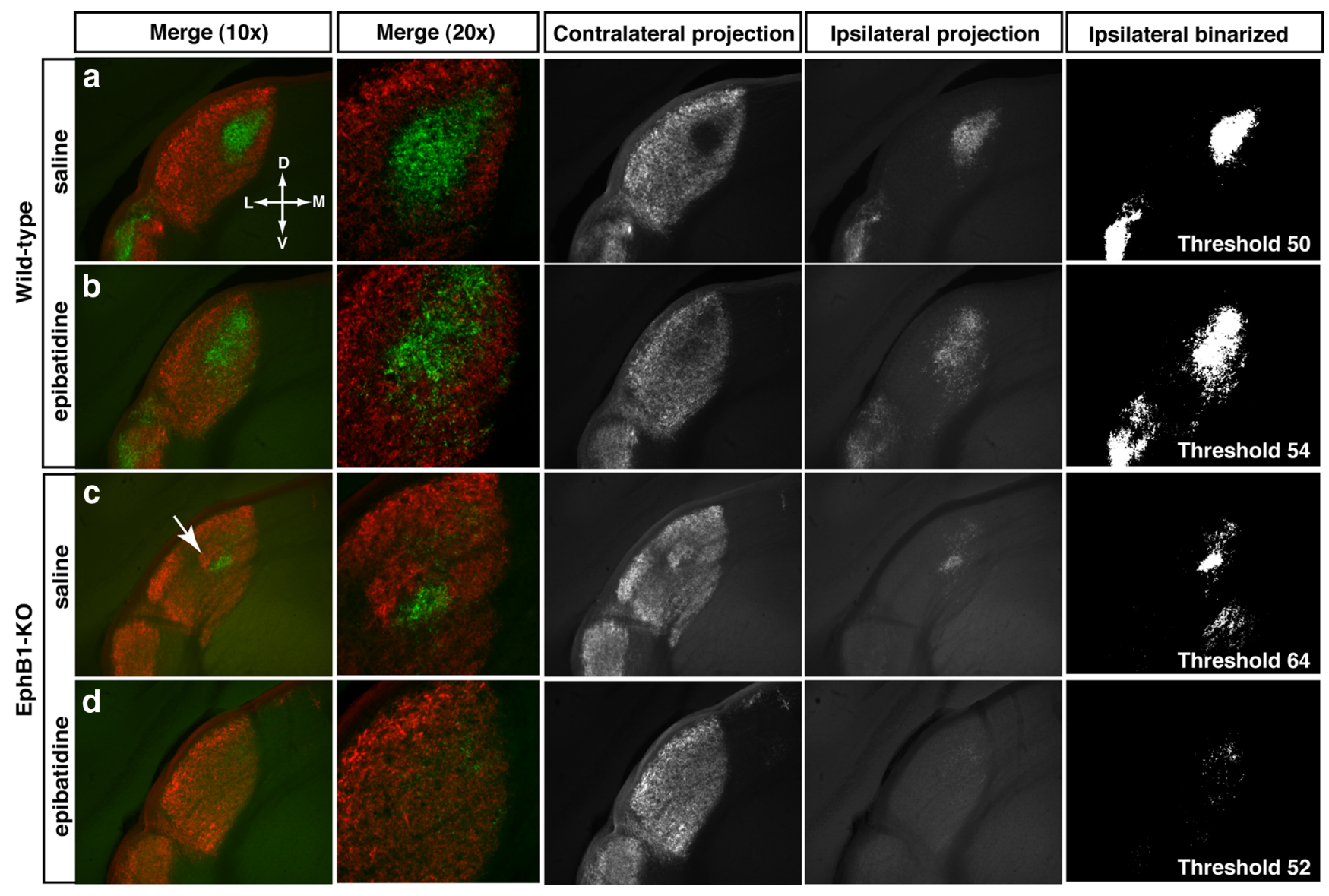

Figure 4. Retinogeniculate projections at P31 after binocular epibatidine treatment from P3 to P6. In wild-type $(\boldsymbol{a}, \boldsymbol{b})$ and $E$ phB1 ${ }^{-1-}(\boldsymbol{c}, \boldsymbol{d})$ mice, ipsilateral fibers are more scattered within the $\mathrm{dLGN}$, cover an extended region of the dLGN, and overlap more with contralateral fibers after epibatidine treatment. In addition, aggregation of the abnormal contralateral patch adjacent to the ipsilateral patch and the optic tract is abolished after epibatidine treatment in EphB1 ${ }^{-1-}$ mice (c, arrow; compare with $\boldsymbol{d}$ ). D, Dorsal; V, ventral; M, medial; L, lateral.

dLGN (Fig. 5b), suggesting that blocking retinal waves results in a reduced number of ipsilateral terminals that are scattered over a larger region of the dLGN in EphB1 $1^{-1-}$ mice (Fig. $4 d$ ). In addition, epibatidine treatment of $E p h B 1^{-1-}$ mice further impairs the segregation of ipsilateral fibers compared with saline-treated EphB1 $1^{-1-}$ mice (Fig. 5c,d). Importantly, in EphB1 $1^{-1-}$ mice, binocular epibatidine treatment prevents the formation of the ectopic contralateral patch formed by fibers from VT retina that are misrouted contralaterally (Fig. $4 c, d$ ).

In conclusion, normal retinal activity is required for the proper segregation and distribution of ipsilateral fibers within a restricted area of the dLGN. In contrast, retinal waves do not seem to be crucial for determining the proportion of ipsilateral terminals in wild-type mice. Finally, retinal activity is required for the segregation of the ectopic contralateral patch in EphB1 $1^{-1-}$ mice.

\section{Discussion}

Here, we describe how abnormal decussation patterns impact targeting in the mouse dLGN. In gain- and loss-of-function models of the guidance receptor EphB1, misrouted VT RGC axons project to the correct topographic location in the opposite dLGN, in a mirror image of the normal pattern (Fig. 6). However, in EphB1 ${ }^{-1-}$ mice, the misrouted axons segregate from the normal contralateral and ipsilateral cohorts. Furthermore, distribution and segregation of ipsilateral axons are altered in $\mathrm{EphB1} 1^{-1-}$ mice. We also show that binocular blockade of retinal activity perturbs ipsilateral fiber distribution and segregation in a similar manner in wild-type and EphB1 ${ }^{-1-}$ mice. Finally, the segregation of the misrouted fibers in EphB1 ${ }^{-1-}$ mice is dependent on normal retinal activity.

These experiments implicate the existence of molecular determinants for ipsilateral projection targeting independent of those used for axon guidance at the optic chiasm, and provide insight into how the interactions of ipsilateral and contralateral axons, in concert with retinal activity, yield eye-specific retinogeniculate targeting.

Eye-specific targeting of retinal axons does not depend on laterality decisions at the midline

Our results show that misrouted RGC axons innervate the appropriate target according to their retinotopic origin (VT vs non-VT retina) even though axons project to the other side of the midline, in agreement with other studies on midline guidance perturbations. In robo and comm fly mutants (Wolf and Chiba, 2000), astray and belladonna zebrafish mutants (Karlstrom et al., 1996; Fricke et al., 2001), the Siamese cat (Guillery, 1969; Kliot and Shatz, 1985), and achiasmatic sheepdogs (Williams et al., 1994), axons project to the retinotopically appropriate targets despite errors in midline guidance. However, it is unclear how altered projections integrate into the normal circuitry, and how the mutated gene relates to midline and targeting defects in these models. Here, we demonstrate that, despite normal targeting, the misrouted fibers do not intermingle with the normal projections and form a patch clearly segregated from the normal ipsilateral and contralateral projections. 


\section{Molecular determinants of eye-specific targeting}

Our results demonstrate that, when RGC axon laterality is perturbed, VT RGCs still target the appropriate $\mathrm{dLGN}$ regions. This finding supports the notion that RGCs and dLGN cells express molecular cues for eye-specific targeting that are distinct from EphB1. Whereas lamina-specific cues have been identified in the retina (Yamagata and Sanes, 2008) and in the optic tectum (Yamagata and Sanes, 2005), molecules that are differentially expressed in eye-specific layers in the dLGN have not been identified (Kawasaki et al., 2004). EphA and ephrin-A gradients in the retina and dLGN mediate topographic mapping of retinogeniculate projections (Feldheim et al., 1998; Huberman et al., 2005; Pfeiffenberger et al., 2005, 2006) and could participate in eye-specific targeting. However, our results and those of Pfeiffenberger et al. (2006) indicate that RGCs from the same retinotopic origin (e.g., VT retina) target quite different dLGN subregions depending on whether they project to the ipsilateral (central dLGN) or contralateral side (dorsal tip of the dLGN). One possibility is that the VT RGCs that project ipsilaterally or contralaterally locally express different levels of EphAs and ephrin-As even though they are intermingled. Alternatively, if expression is similar in all VT RGCs, molecular labels in addition to EphA-ephrin-A gradients must mediate the differential targeting of VT RGCs and/or these two populations of VT RGCs respond differently to these gradients (for human, see Lambot et al., 2005).

Several genes have been implicated in specification of RGCs but do not appear to directly mediate eye-specific targeting (Pak et al., 2004; Quina et al., 2005; Huberman et al., 2008b, 2009; Kim et al., 2008; Badea et al., 2009). One interesting candidate for the formation of eye-specific projections is Ten_M3, expressed in gradients within the retina and dLGN (Leamey et al., 2007). In knock-outs of Ten_M3, the distribution of ipsilateral fibers is perturbed in the dLGN and binocular vision is defective, but how Ten_M3 mediates eye-specific innervation is unknown.

\section{Disruption of the eye-specific retinogeniculate axon} distribution and segregation in $E p h B 1^{-1-}$ mice

Our results indicate that, even under conditions of normal activity, segregation of ipsilateral fibers in the $E \mathrm{phB1} 1^{-/-}$mice is perturbed and correlates with the reduction in the number of ipsilaterally projecting VT RGCs (Dräger and Olsen, 1980; Williams et al., 2003). One explanation of this finding is that the reduction of ipsilateral fibers impairs activity-based competition and leads to altered segregation, with contralateral fibers remaining in the ipsilateral territory when ipsilateral RGC axons are in the minority. Similar effects were found after an imbalance in the activity of eye-specific inputs after
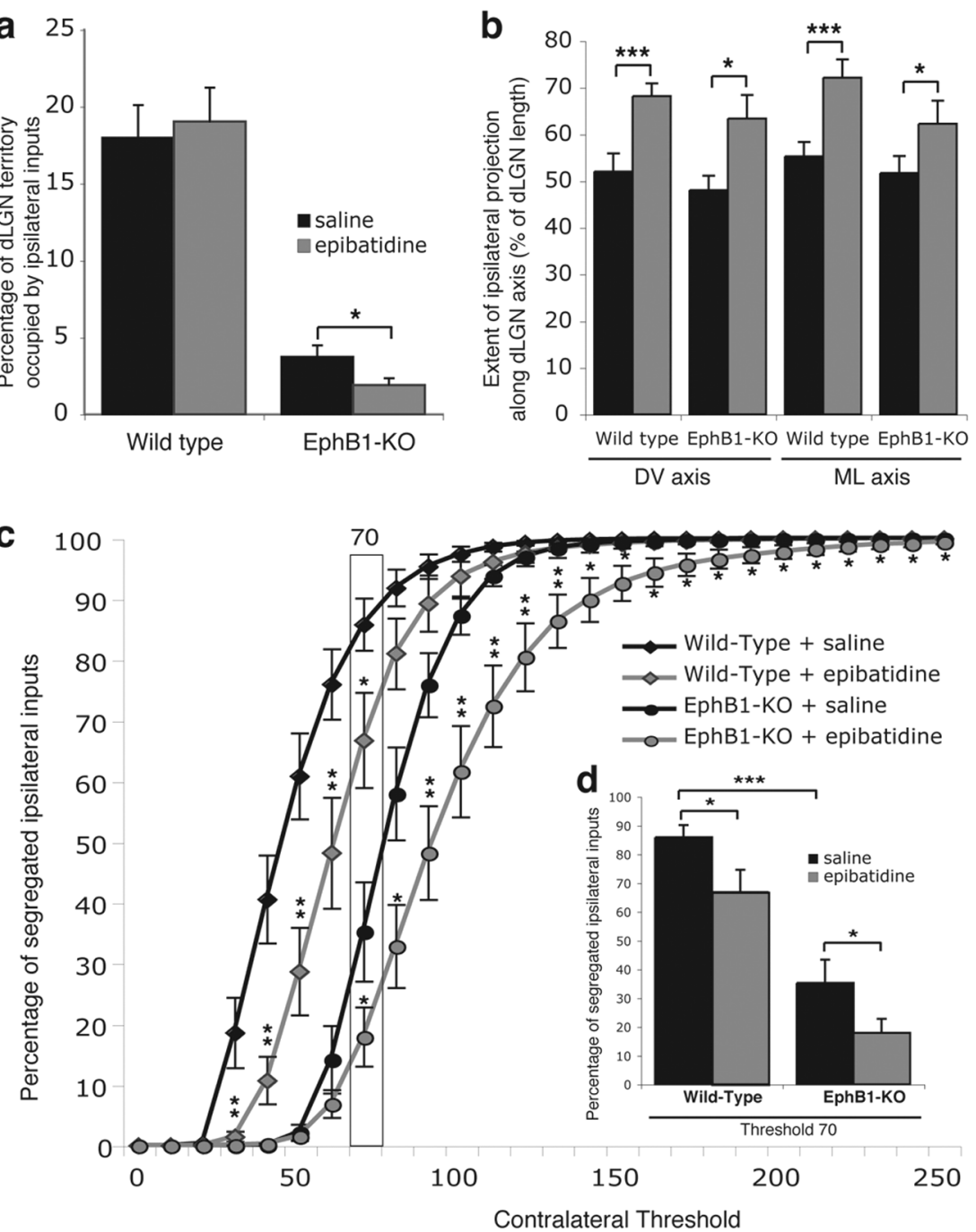

Figure 5. Comparison of ipsilateral projections in wild-type and EphB1 ${ }^{-1-}$ mice after epibatidine treatment. $\boldsymbol{a}$, Binocular epibatidine treatment reduced the percentage of $\mathrm{dLGN}$ occupied by ipsilateral fibers in EphB1 ${ }^{-/-}$mice but had no effect on

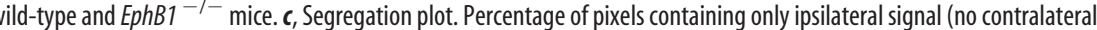

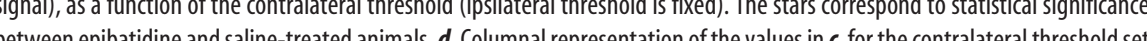
at 70 . Segregation of ipsilateral axons in saline treated $E p h B 1^{-1-}$ mice is significantly impaired compared with wild-type mice, similar to the differences seen in nontreated animals. Segregation of ipsilateral fibers is further perturbed in the dLGN of both wild-type and EphB1 ${ }^{-1-}$ mice after epibatidine treatment. Error bars indicate SEM values. ${ }^{*} p<0.05,{ }^{* *} p<0.01,{ }^{* * *} p<0.001$.

monocular injection of epibatidine (Penn et al., 1998) or elevation of cAMP levels (Stellwagen and Shatz, 2002). These results strengthen the idea that competition between different axonal populations sculpts final targeting and axon arbor extension (Guillery, 1988). It is also possible that, because EphBs and ephrin-Bs are important for synaptogenesis, directional branch extension, and arborization (Hindges et al., 2002; McLaughlin et al., 2003; Kayser et al., 2008; Lim et al., 2008), the impaired eye-specific segregation in EphB1 ${ }^{-1-}$ mice arises from defects in the refinement process such as in pruning of exuberant branches, directional arborization, or weakened synapses.

Misrouted RGC axons segregate into patches: a molecular and/or activity-dependent process?

The most striking result of this study is the formation in EphB1 $1^{-1-}$ mice of an ectopic patch by VT axons that aberrantly cross the midline. This patch of misrouted axons is positioned next to the zone receiving the ipsilateral projection and is remi- 

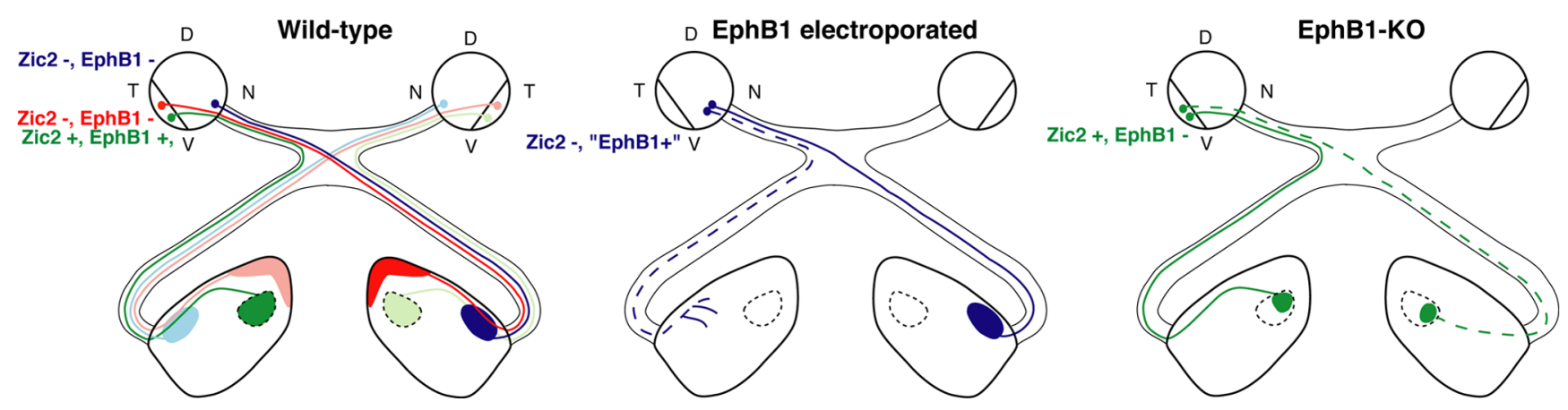

Figure 6. The retinogeniculate pathway in mouse models with impaired decussation at the optic chiasm. In wild-type mice, nasal RGCs (Zic2-/EphB1-; blue) project contralaterally to the lateral part of the dLGN. VT RGCs (Zic2 +/EphB1 + ; green) project ipsilaterally to the dorsomedial part of the dLGN and later-born VT RGCs (Zic2 - /EphB1 -; red) project contralaterally to the dorsal tip of the dLGN. Ectopic EphB1 expression [Zic2 - /EphB1+] in non-VT RGCs leads to the misrouting of some RGC fibers ipsilaterally (blue dashed line), which still target the contralateral-recipient region of the dLGN. In EphB1 ${ }^{-1-}$ mice, some VT RGCS (Zic2+/EphB1-) project contralaterally to the dorsomedial part of the dLGN (green dashed line), in a mirror image of the normal ipsilateral projection. D, Dorsal; $\mathrm{V}$, ventral; $\mathrm{T}$, temporal; $\mathrm{N}$, nasal.

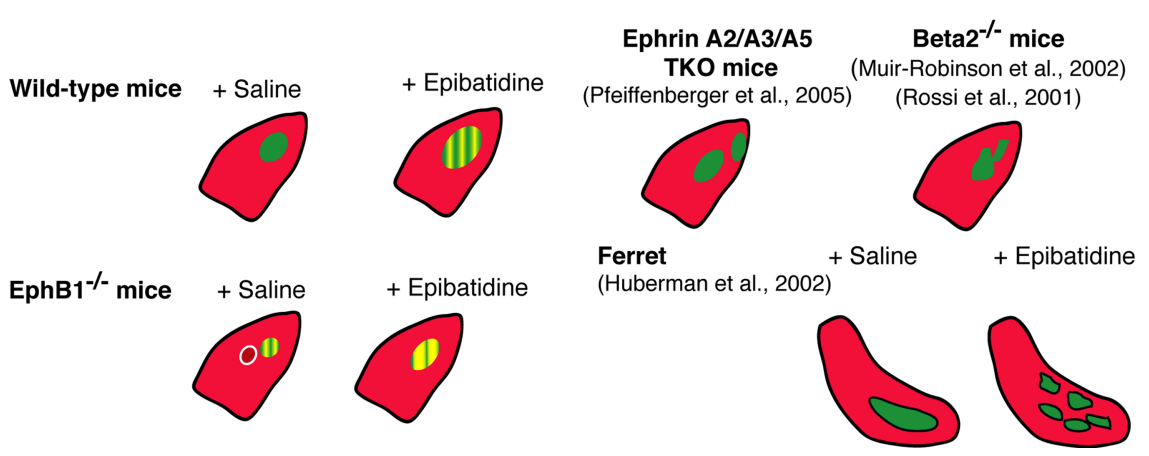

Figure 7. Schematic representation of eye-specific projection patterns to the dLGN. Retinogeniculate projections from the contralateral eye (red) and ipsilateral eye (green) in different mouse and ferret models. The extent of impairment in eye-specific segregation is represented as a grating of green and yellow, in proportion to the observed overlap.

niscent of the segregation of retinal axons when an extra eye is transplanted into a tadpole (Constantine-Paton and Law, 1978). In a Hebbian model of synaptic reinforcement and weakening (Butts et al., 2007), misrouted VT retinal axons in EphB1 ${ }^{-1-}$ mice would segregate from other projections when their activity is uncorrelated, if they have a different origin, or project from the other eye, as is the case here. Indeed, the normal contralateral fibers surrounding misrouted axons do not arise from the VT region of the retina, reducing the chance of correlated activity between these two sets of inputs. Another possibility is that the ipsilateral and contralateral VT RGCs could express different surface molecules that would facilitate their fasciculation and terminal aggregation and prevent the mingling with different retinal fiber types. The expression of these adhesion molecules could be regulated by activity (Itoh et al., 1995; Hanson and Landmesser, 2004) and explain why the patch disappears after blockade of retinal correlated activity during the first postnatal week.

\section{Segregation of ipsilateral axons in the mature dLGN requires correlated activity in the retina}

We analyzed the role of retinal activity on eye-specific refinement in both wild-type and EphB1 ${ }^{-1-}$ mice and found that binocular blockade of retinal waves during the first postnatal week perturbs ipsilateral fiber distribution (ipsilateral fibers are more scattered and extend over a larger area within the dLGN) and segregation (greater overlap of ipsilateral and contralateral fibers) in the dLGN. These features are more severely disrupted in EphB1 $1^{-1-}$ mice (Fig. 7), because the refinement is perturbed in this mutant even without epibatidine treatment.
We describe for the first time the longterm effects of binocular epibatidine treatment during the first postnatal week on eyespecific refinement in mouse dLGN. Our results contradict previous reports on the long-term changes in the ipsilateral projection in ferrets treated with epibatidine (Huberman et al., 2002) and in mice that lack the $\beta 2$ subunit of the nicotinic acetylcholine receptor (beta2 $2^{-1-}$ mice) and thus cholinergic-dependent retinal waves of activity (Bansal et al., 2000). In these studies, ipsilateral fibers were seen to occupy a greater percentage of the dLGN but display normal eye-specific segregation (Rossi et al., 2001; Huberman et al., 2002; Muir-Robinson et al., 2002) (Fig. 7). For beta $2^{-1-}$ mice, the most likely explanation is that pharmacological treatment with epibatidine is not equivalent to the genetic removal of the $\beta 2$ receptor subunit. Indeed, a recent report questions the reported absence of retinal waves in beta $2^{-1-}$ mice (Sun et al., 2008a), whereas there is a consensus on the effect of epibatidine treatment on retinal waves (Cang et al., 2005; Sun et al., 2008 b). The different effects of epibatidine treatment on retinogeniculate projections in mice and ferrets may simply reflect species-specific effects as LGN structure is laminated in ferrets and thus more complex.

The exact features of retinal activity necessary for eye-specific segregation are under debate (Chalupa, 2009; Feller, 2009), and further studies on mouse models with more specific perturbations in retinal activity should resolve this issue.

\section{How does activity function to direct RGC innervation of the dLGN?}

Our experiments highlight the interplay between molecular determinants and activity during targeting (Sanes and Yamagata, 2009). Several activity-regulated molecules (intriguingly related to the immune system), such as $\mathrm{MHCI}, \mathrm{Clq}$, and pentraxins, are required for the refinement of eye-specific projections (Huh et al., 2000; Bjartmar et al., 2006; Stevens et al., 2007). Retinal activity could affect the response of RGCs by changing gene expression or localization of such molecules to yield proper retinogeniculate fiber distribution and segregation (Nicol et al., 2007). Thus, molecular specification and retinal activity likely work in concert to perfect the organization of RGC projections in their target. 


\section{References}

Badea TC, Cahill H, Ecker J, Hattar S, Nathans J (2009) Distinct roles of transcription factors brn3a and brn3b in controlling the development, morphology, and function of retinal ganglion cells. Neuron 61:852-864.

Bansal A, Singer JH, Hwang BJ, Xu W, Beaudet A, Feller MB (2000) Mice lacking specific nicotinic acetylcholine receptor subunits exhibit dramatically altered spontaneous activity patterns and reveal a limited role for retinal waves in forming $\mathrm{ON}$ and OFF circuits in the inner retina. J Neurosci 20:7672-7681.

Beg AA, Sommer JE, Martin JH, Scheiffele P (2007) alpha2-Chimaerin is an essential EphA4 effector in the assembly of neuronal locomotor circuits. Neuron 55:768-778.

Bjartmar L, Huberman AD, Ullian EM, Rentería RC, Liu X, Xu W, Prezioso J, Susman MW, Stellwagen D, Stokes CC, Cho R, Worley P, Malenka RC, Ball S, Peachey NS, Copenhagen D, Chapman B, Nakamoto M, Barres BA, Perin MS (2006) Neuronal pentraxins mediate synaptic refinement in the developing visual system. J Neurosci 26:6269-6281.

Butts DA, Kanold PO, Shatz CJ (2007) A burst-based "Hebbian" learning rule at retinogeniculate synapses links retinal waves to activity-dependent refinement. PLoS Biol 5:e61.

Cang J, Rentería RC, Kaneko M, Liu X, Copenhagen DR, Stryker MP (2005) Development of precise maps in visual cortex requires patterned spontaneous activity in the retina. Neuron 48:797-809.

Chalupa LM (2007) A reassessment of the role of activity in the formation of eye-specific retinogeniculate projections. Brain Res Rev 55:228-236.

Chalupa LM (2009) Retinal waves are unlikely to instruct the formation of eye-specific retinogeniculate projections. Neural Dev 4:25.

Constantine-Paton M, Law MI (1978) Eye-specific termination bands in tecta of three-eyed frogs. Science 202:639-641.

Dräger UC, Olsen JF (1980) Origins of crossed and uncrossed retinal projections in pigmented and albino mice. J Comp Neurol 191:383-412.

Feldheim DA, Vanderhaeghen P, Hansen MJ, Frisén J, Lu Q, Barbacid M, Flanagan JG (1998) Topographic guidance labels in a sensory projection to the forebrain. Neuron 21:1303-1313.

Feller MB (2009) Retinal waves are likely to instruct the formation of eyespecific retinogeniculate projections. Neural Dev 4:24.

Fricke C, Lee JS, Geiger-Rudolph S, Bonhoeffer F, Chien CB (2001) astray, a zebrafish roundabout homolog required for retinal axon guidance. Science 292:507-510.

García-Frigola C, Carreres MI, Vegar C, Herrera E (2007) Gene delivery into mouse retinal ganglion cells by in utero electroporation. BMC Dev Biol 7:103.

García-Frigola C, Carreres MI, Vegar C, Mason C, Herrera E (2008) Zic2 promotes axonal divergence at the optic chiasm midline by EphB1dependent and -independent mechanisms. Development 135:1833-1841.

Godement P, Salaün J, Imbert M (1984) Prenatal and postnatal development of retinogeniculate and retinocollicular projections in the mouse. J Comp Neurol 230:552-575.

Guillery RW (1969) An abnormal retinogeniculate projection in Siamese cats. Brain Res 14:739-741.

Guillery RW (1988) Competition in the development of the visual pathways. In: The making of the nervous system (Parnavelas JG, Stern CD, Stirling RV, eds), pp 356-379. Oxford: Oxford UP.

Hanson MG, Landmesser LT (2004) Normal patterns of spontaneous activity are required for correct motor axon guidance and the expression of specific guidance molecules. Neuron 43:687-701.

Herrera E, Brown L, Aruga J, Rachel RA, Dolen G, Mikoshiba K, Brown S, Mason CA (2003) Zic2 patterns binocular vision by specifying the uncrossed retinal projection. Cell 114:545-557.

Hindges R, McLaughlin T, Genoud N, Henkemeyer M, O’Leary DD (2002) EphB forward signaling controls directional branch extension and arborization required for dorsal-ventral retinotopic mapping. Neuron 35:475-487.

Huberman AD, Stellwagen D, Chapman B (2002) Decoupling eye-specific segregation from lamination in the lateral geniculate nucleus. J Neurosci 22:9419-9429.

Huberman AD, Murray KD, Warland DK, Feldheim DA, Chapman B (2005) Ephrin-As mediate targeting of eye-specific projections to the lateral geniculate nucleus. Nat Neurosci 8:1013-1021.

Huberman AD, Feller MB, Chapman B (2008a) Mechanisms underlying development of visual maps and receptive fields. Annu Rev Neurosci 31:479-509.
Huberman AD, Manu M, Koch SM, Susman MW, Lutz AB, Ullian EM, Baccus SA, Barres BA (2008b) Architecture and activity-mediated refinement of axonal projections from a mosaic of genetically identified retinal ganglion cells. Neuron 59:425-438.

Huberman AD, Wei W, Elstrott J, Stafford BK, Feller MB, Barres BA (2009) Genetic identification of an on-off direction-selective retinal ganglion cell subtype reveals a layer-specific subcortical map of posterior motion. Neuron 62:327-334.

Huh GS, Boulanger LM, Du H, Riquelme PA, Brotz TM, Shatz CJ (2000) Functional requirement for class I MHC in CNS development and plasticity. Science 290:2155-2159.

Itoh K, Stevens B, Schachner M, Fields RD (1995) Regulated expression of the neural cell adhesion molecule L1 by specific patterns of neural impulses. Science 270:1369-1372.

Iwasato T, Katoh H, Nishimaru H, Ishikawa Y, Inoue H, Saito YM, Ando R, Iwama M, Takahashi R, Negishi M, Itohara S (2007) Rac-GAP alphachimerin regulates motor-circuit formation as a key mediator of EphrinB3/ EphA4 forward signaling. Cell 130:742-753.

Jaubert-Miazza L, Green E, Lo FS, Bui K, Mills J, Guido W (2005) Structural and functional composition of the developing retinogeniculate pathway in the mouse. Vis Neurosci 22:661-676.

Jen JC, Chan WM, Bosley TM, Wan J, Carr JR, Rüb U, Shattuck D, Salamon G, Kudo LC, Ou J, Lin DD, Salih MA, Kansu T, Al Dhalaan H, Al Zayed Z, MacDonald DB, Stigsby B, Plaitakis A, Dretakis EK, Gottlob I, et al. (2004) Mutations in a human ROBO gene disrupt hindbrain axon pathway crossing and morphogenesis. Science 304:1509-1513.

Kaas JH (2005) Serendipity and the Siamese cat: the discovery that genes for coat and eye pigment affect the brain. ILAR J 46:357-363.

Karlstrom RO, Trowe T, Klostermann S, Baier H, Brand M, Crawford AD, Grunewald B, Haffter P, Hoffmann H, Meyer SU, Müller BK, Richter S, van Eeden FJ, Nüsslein-Volhard C, Bonhoeffer F (1996) Zebrafish mutations affecting retinotectal axon pathfinding. Development 123: 427-438.

Kawasaki H, Crowley JC, Livesey FJ, Katz LC (2004) Molecular organization of the ferret visual thalamus. J Neurosci 24:9962-9970.

Kayser MS, Nolt MJ, Dalva MB (2008) EphB receptors couple dendritic filopodia motility to synapse formation. Neuron 59:56-69.

Kim IJ, Zhang Y, Yamagata M, Meister M, Sanes JR (2008) Molecular identification of a retinal cell type that responds to upward motion. Nature 452:478-482.

Kliot M, Shatz CJ (1985) Abnormal development of the retinogeniculate projection in Siamese cats. J Neurosci 5:2641-2653.

Kullander K, Butt SJ, Lebret JM, Lundfald L, Restrepo CE, Rydström A, Klein R, Kiehn O (2003) Role of EphA4 and EphrinB3 in local neuronal circuits that control walking. Science 299:1889-1892.

Lambot MA, Depasse F, Noel JC, Vanderhaeghen P (2005) Mapping labels in the human developing visual system and the evolution of binocular vision. J Neurosci 25:7232-7237.

Leamey CA, Merlin S, Lattouf P, Sawatari A, Zhou X, Demel N, Glendining KA, Oohashi T, Sur M, Fässler R (2007) Ten_M3 regulates eye-specific patterning in the mammalian visual pathway and is required for binocular vision. PLoS Biol 5:e241.

Lee R, Petros TJ, Mason CA (2008) Zic2 regulates retinal ganglion cell axon avoidance of ephrinB2 through inducing expression of the guidance receptor EphB1. J Neurosci 28:5910-5919.

Lim BK, Matsuda N, Poo MM (2008) Ephrin-B reverse signaling promotes structural and functional synaptic maturation in vivo. Nat Neurosci $11: 160-169$.

McLaughlin T, Hindges R, Yates PA, O’Leary DD (2003) Bifunctional action of ephrin-B1 as a repellent and attractant to control bidirectional branch extension in dorsal-ventral retinotopic mapping. Development 130:2407-2418.

Muir-Robinson G, Hwang BJ, Feller MB (2002) Retinogeniculate axons undergo eye-specific segregation in the absence of eye-specific layers. J Neurosci 22:5259-5264.

Nicol X, Voyatzis S, Muzerelle A, Narboux-Nême N, Südhof TC, Miles R, Gaspar P (2007) cAMP oscillations and retinal activity are permissive for ephrin signaling during the establishment of the retinotopic map. Nat Neurosci 10:340-347.

Pak W, Hindges R, Lim YS, Pfaff SL, O’Leary DD (2004) Magnitude of binocular vision controlled by islet- 2 repression of a genetic program that specifies laterality of retinal axon pathfinding. Cell 119:567-578. 
Penn AA, Riquelme PA, Feller MB, Shatz CJ (1998) Competition in retinogeniculate patterning driven by spontaneous activity. Science 279:21082112.

Petros TJ, Shrestha BR, Mason C (2009) Specificity and sufficiency of EphB1 in driving the ipsilateral retinal projection. J Neurosci 29:3463-3474.

Pfeiffenberger C, Cutforth T, Woods G, Yamada J, Rentería RC, Copenhagen DR, Flanagan JG, Feldheim DA (2005) Ephrin-As and neural activity are required for eye-specific patterning during retinogeniculate mapping. Nat Neurosci 8:1022-1027.

Pfeiffenberger C, Yamada J, Feldheim DA (2006) Ephrin-As and patterned retinal activity act together in the development of topographic maps in the primary visual system. J Neurosci 26:12873-12884.

Quina LA, Pak W, Lanier J, Banwait P, Gratwick K, Liu Y, Velasquez T, O’Leary DD, Goulding M, Turner EE (2005) Brn3a-expressing retinal ganglion cells project specifically to thalamocortical and collicular visual pathways. J Neurosci 25:11595-11604.

Reese BE, Cowey A (1987) The crossed projection from the temporal retina to the dorsal lateral geniculate nucleus in the rat. Neuroscience 20: 951-959.

Rossi FM, Pizzorusso T, Porciatti V, Marubio LM, Maffei L, Changeux JP (2001) Requirement of the nicotinic acetylcholine receptor beta $2 \mathrm{sub}-$ unit for the anatomical and functional development of the visual system. Proc Natl Acad Sci U S A 98:6453-6458.

Sanes JR, Yamagata M (2009) Many paths to synaptic specificity. Annu Rev Cell Dev Biol 25:161-195.

Stellwagen D, Shatz CJ (2002) An instructive role for retinal waves in the development of retinogeniculate connectivity. Neuron 33:357-367.

Stevens B, Allen NJ, Vazquez LE, Howell GR, Christopherson KS, Nouri N, Micheva KD, Mehalow AK, Huberman AD, Stafford B, Sher A, Litke AM, Lambris JD, Smith SJ, John SW, Barres BA (2007) The classical complement cascade mediates CNS synapse elimination. Cell 131:1164-1178.

Sun C, Warland DK, Ballesteros JM, van der List D, Chalupa LM (2008a) Retinal waves in mice lacking the beta2 subunit of the nicotinic acetylcholine receptor. Proc Natl Acad Sci U S A 105:13638-13643.

Sun C, Speer CM, Wang GY, Chapman B, Chalupa LM (2008b) Epibatidine application in vitro blocks retinal waves without silencing all retinal ganglion cell action potentials in developing retina of the mouse and ferret. J Neurophysiol 100:3253-3263.

Torborg CL, Feller MB (2004) Unbiased analysis of bulk axonal segregation patterns. J Neurosci Methods 135:17-26.

Victor JD, Apkarian P, Hirsch J, Conte MM, Packard M, Relkin NR, Kim KH, Shapley RM (2000) Visual function and brain organization in nondecussating retinal-fugal fibre syndrome. Cereb Cortex 10:2-22.

Wegmeyer H, Egea J, Rabe N, Gezelius H, Filosa A, Enjin A, Varoqueaux F, Deininger K, Schnütgen F, Brose N, Klein R, Kullander K, Betz A (2007) EphA4-dependent axon guidance is mediated by the RacGAP alpha2chimaerin. Neuron 55:756-767.

Williams RW, Hogan D, Garraghty PE (1994) Target recognition and visual maps in the thalamus of achiasmatic dogs. Nature 367:637-639.

Williams SE, Mann F, Erskine L, Sakurai T, Wei S, Rossi DJ, Gale NW, Holt CE, Mason CA, Henkemeyer M (2003) Ephrin-B2 and EphB1 mediate retinal axon divergence at the optic chiasm. Neuron 39:919-935.

Williams SE, Grumet M, Colman DR, Henkemeyer M, Mason CA, Sakurai T (2006) A role for Nr-CAM in the patterning of binocular visual pathways. Neuron 50:535-547.

Wolf BD, Chiba A (2000) Axon pathfinding proceeds normally despite disrupted growth cone decisions at CNS midline. Development 127:20012009.

Wong WT, Myhr KL, Miller ED, Wong RO (2000) Developmental changes in the neurotransmitter regulation of correlated spontaneous retinal activity. J Neurosci 20:351-360.

Yamagata M, Sanes JR (2005) Versican in the developing brain: laminaspecific expression in interneuronal subsets and role in presynaptic maturation. J Neurosci 25:8457-8467.

Yamagata M, Sanes JR (2008) Dscam and Sidekick proteins direct laminaspecific synaptic connections in vertebrate retina. Nature 451:465-469.

Zhou ZJ, Zhao D (2000) Coordinated transitions in neurotransmitter systems for the initiation and propagation of spontaneous retinal waves. J Neurosci 20:6570-6577. 